\title{
PREFACE
}

\section{A SPECIAL SELECTION ON BIOMECHANICS IN MEDICAL APPLICATIONS - PART I}

Part I of this special issue sums up the selections focusing on the Applications of Biomechanics in Medicine. The details of the accepted 20 papers are as follows,

Guanghui $\mathrm{Wu}$ et al. establish an ovine model for $\mathrm{CH}-\mathrm{VAD}$ (CH Biomedical Inc., JiangSu, China- Ventricular Assist Device) implantable ventricular assist device (IVAD) to evaluate experimental protocols. IVAD is implanted into the chest of sheep without stopping the beating heart through a left thoracotomy, and the inflow cannula is connected to the left ventricular apex and the outflow cannula is anastomosed to the descending aorta. Sheep models suitable for implantation of $\mathrm{CH}-$ VAD implantable LVAD are successfully established and the appropriate safety evaluation indicators of this model are validated in the course of the animal experiments and the survival rate of the experiment are improved gradually over time.

Je-Nam Kim et al. propose a health monitoring system for knee joints, which is able to classify lower extremity movements using the angle and acceleration components of these joints with classification algorithm. The proposed monitoring system consists of a wearable frame placed on the knee joint and a sensor part for monitoring the knee joint angle and acceleration, and a wireless communication part for transferring bio signals to a smart device. This study shows that the monitoring system detects postures that negatively affect knee joints and informs a user when these postures are adopted, thereby helping to maintain healthy knee joints.

Monan Wang et al. build a simulation model of fracture healing process and describe the relationship among mechanical stability, oxygen levels in tissues and tissue differentiation during the second fracture healing according to the mechanical conditions of fracture fixation and the oxygen levels in the tissues. They take the three-dimensional model as the research object, solve the mechanical stimulation by finite element method, establish the partial differential equation to solve the spatial and temporal variation of the oxygen in tissues. The simulation program of fracture healing process is independently developed in Eclipse environment, and the 
simulation results are compared with experimental data to verify that oxygen and interosseous stability are both important factors affecting fracture healing.

Lung-Fa Pan et al. optimize the minimum detectable difference (MDD) of a cardiac X-ray imaging system using the Taguchi L8(27) analysis and a precise line pair gauge. The optimal combination of the four critical factors of the cardiac X-ray imaging system, namely X-ray focus, kilovoltage ( $\mathrm{kVp})$, milliamper-seconds (mAs), and source image distance (SID), providing the MDD is calculated via the Taguchi analysis and experimentally verified. The Taguchi L8 analysis is proved to be instrumental in optimizing the cardiac X-ray imaging system MDD and is recommended to be used jointly with the revised Student's $t$-test analysis for improving the spatial resolution of instant X-ray images.

Gwang Moon Eom et al. aim to compare the ankle joint moments in difference foot strike patterns during stair descent. Subjects perform two trials of descending stairs in rearfoot strike (RFS) and forefoot strike (FFS) strategies. Kinematic and kinetic data are measured by a motion capture system and force plates. Ankle joint moments, ground reaction forces, and moment arms in three planes of motion are calculated from the measured data. The result indicates RFS would be a better strategy than FFS for stair descent in terms of ankle joint load. It suggests that the RFS could be a better strategy to reduce injuries and diseases in ankle joint that may caused by repetitive loading.

Aili $\mathrm{Qu}$ et al. investigate effects of material model selection on the responses of lateral impact simulations and construct a seating pelvic complex model. The model is subjected to a series of impacts at velocity of $3-10 \mathrm{~m} / \mathrm{s}$, and two material models are respectively assigned to the pelvic bone to evaluate the accuracy of the simulation. The results show that the pelvic response and fracture pattern with plastic elastic material model agreed well with the literature, while linear elastic material model is dissatisfied, and drastic change of arterial pressure is responsible for hemorrhages associated with pelvic fracture. Therefore, the plastic elastic material model is recommended in the pelvic complex impact simulation.

Jae-Hoon Heo et al. test the effect of sensory electrical stimulation on resting tremors in patients with PD (Parkinson's disease) and SWEDDS (dopaminergic deficits). Three wrist muscles are stimulated for $15 \mathrm{~s}$ using SES (Sensory electrical stimulation) with a stimulation intensity lower than the motor threshold. Angular motion of the index finger is measured via a tri-axial gyrosensor before, during, and after stimulation. Outcome measures include the amplitude and peak frequency of the angular motion of the index finger. It shows that SES suppressed tremors in patients with PD (Parkinson's disease), but not with SWEDDs. The results could help understand the pathophysiological differences of tremor between PD and SWEDDs.

Tian $\mathrm{Xu}$ et al. present three kinds of collision reaction strategies for increasing safety between human and robot interaction without relying on torque sensors. In the proposed algorithms, motor torque is estimated by driver current. Experiments are tested between a 1-DOF manipulator system and a human being to verify 
efficiency of the proposed algorithms. This reaction strategy makes the robot act like a soft spring which can reduce impact effect to increase human-robot interaction safety. These proposed algorithms would be applied to serial manipulator with more degrees of freedom in the future.

Jingang Jiang et al. develop a medical device that can assist doctors in prostate scans and biopsy puncture. Based on the posture adjustment mechanism, a passive interlocking transrectal ultrasound probe position and posture adjustment mechanism capable of assisting the doctor in prostate scanning and puncture intervention is designed. The overall configuration of the mechanism is divided into a 3-degree of freedom (DOF) position adjustment module, 2-DOF posture adjustment module, and 2-DOF ultrasonic probe rotation and feed module. The results show that the ultrasonic probe can achieve reliable locking and effectively meet the operation requirements.

Junghyuk Ko et al. study muscle strength and the ability to generate rapid torque for knee joint in elderly men and women. Healthy elderly participants (10 men and 10 women) perform maximal voluntary knee extension and flexion during concentric, isometric and eccentric conditions. Independent $t$-tests are employed in the comparison of elderly women with elderly men. Elderly women exhibit weaker isometric flexion and eccentric extension strength compared to elderly men $(p<0.05)$. These results indicate that the deteriorated rate of torque development (RTD) as well as muscle strength per body mass may be associated with a higher frequency of falls in elderly women than in elderly men. This study suggests that training of specific-contraction type should be considered for fall prevention in elderly women.

Yanyan Chen et al. apply trajectory linearization control (TLC) to design an autonomous nonlinear trajectory tracking controller for a novel rehabilitation exoskeleton shoulder joint. As a new application in the exoskeleton shoulder joint controller design, the controller in this research contains two loops that separately based on the inverse kinematics and pseudo-inverse dynamics models of the exoskeleton shoulder. Two PI controllers as the error regulator can reduce the tracking error. The simulation of three different trajectories for single DOF movement of shoulder joint (shoulder flexion and extension, abduction and extension), and the movement of both the two degrees are given. The simulation results show that the TLC controller can follow the exoskeleton shoulder trajectory steadily and accurately.

Qiudong $\mathrm{Yu}$ et al. address a customized post-and-core design for the posterior tooth implant. Teeth, root canal, post-and-core with different implant lengths in five masticatory directions are analyzed with finite element analysis to evaluate the stress distribution. The results suggest that, with the similar shape of the post-andcore structure and the root canal, the tooth stress trend is uniform. When the length of the post-and-core structure $\mathrm{L}$ is $0 \mathrm{~mm}$, i.e., two thirds of the root canal length, the root canal stress is the minimum. Therefore, the customized design of the post-and-core structure could well adapt to any kind of root canal, and the length of 
the post-and-core structure $L$ provides guidance for the post-and-core crown prosthesis in clinic.

Tianjiao Zheng et al. explore a lower-limb exoskeleton for walking assistance in a compact and convenient approach. In this exoskeleton, a modular structure and actuator via steel cables are specially designed. A distributed control system based on DSP (TMS320F28335, $150 \mathrm{Mz}$ ) and ARM (STM32F103, $72 \mathrm{Mz}$ ) is also employed. The control-method architecture with three stages is presented for gait generation of the exoskeleton. Four basic states and seven intended motions with reference trajectories are designed. The results verify the effectiveness of the system design and the gait-generation approach proposed in this paper. The overall exoskeleton system is compact, and the corresponding operations are convenient and reliable. In the future work, they will research the gait planning method for stability based on this exoskeleton.

Fan Peng et al. demonstrate that activated astrocytes under high intraocular pressure (IOP) play an important role in compression of optic nerve and block of axonal transport. Four-week duration of ocular hypertension (more than $20 \mathrm{~mm} \mathrm{Hg}$ ) rats induced by cauterizing of three episcleral vessels and administering a fluorouracil subconjunctival injection in the right eye are enrolled and the left eyes of all the rats are used as a self-control. It can be concluded that the activated astrocytes might squeeze the optic nerve, likely leading to optic nerve distortion and axonal flow blockage. They will research the changes in axonal transport and the morphology of astrocytes at different times of IOP maintenance in future work.

Jin Wan Seok et al. use a subminiature ultrasonic transducer with a miniaturized parametric speaker to overcome hearing loss. The transducer generates sound waves with high directionality. These sound waves are focused on the umbo located the center of the tympanic membrane and connected to ossicles of the middle ear. To generate sound waves, various parameters are considered, such as target distance, radiation area and primary frequency. The directionality of the proposed transducer using extracted parameters at audible frequencies is tested. The results confirm high directionality and audible sound generated by the proposed transducer. The method can be expected to be applied to high-efficiency hearing rehabilitation devices and various multimedia devices.

Yu-Chen Yen et al. research Biokinetic model of Tc-99m-Methoxy-isobutylisonitrile (MIBI) for eight patients undergone myocardial perfusion examination using gamma camera and MATLAB program. A six-compartment model is adopted to interpret the metabolic mechanism of each patient. A dimensionless AT index of disagreement between the experimental data and MATLAB optimal solution - is proposed of validating the applied acquisition system and analytical method feasibility. The proposed MATLAB-based fitting of in vivo data with the theoretical results is instrumental for assessing the radiation dose received by the Tc-99m MIBI scan participants. It is recommended to slightly modify the standard protocol for patient undergone myocardial perfusion examination, to satisfy the ALARA (As Low as Reasonably Approach) princ 
Gwang Moon Eom et al. study a foot strike strategy that can reduce the ankle joint load during stair ascent by comparing the ankle joint load in two strategies of initial contact during stair ascent. Twenty young subjects perform ascending stairs with two strategies, i.e., RFS and FFS. Kinematic data is measured from 12 cameras and the ground reaction force is measured by a force plate inserted in the second step of four-step stairs. Four ankle related kinetic variables are derived from the measured data, i.e., joint reaction force, moment and the magnitude and moment arm of ground reaction force. The results suggest that RFS would be a strategy to reduce ankle joint loads during stair ascent.

Monan Wang et al. discuss a collision detection algorithm of bounding volume hierarchy based on virtual sphere to further improve the efficiency and stability of collision detection. The proposed algorithm is applied to two large and mediumsized virtual scenes to implement the collision detection between the vastus lateralis muscle, thigh and surgical instrument. Based on the virtual sphere, the collision detection algorithm of bounding volume hierarchy can implement efficient and stable collision detection in a virtual surgery system. In future work, the collision detection algorithm will be combined based on the virtual sphere with the virtual cutting algorithm and the soft tissue deformation algorithm for more test analysis.

Woo Suk Chong et al. apply a foldable design to an electric wheelchair to improve its mobility and portability and use carbon composite to the body frame to lighten the wheelchair and increase the convenience of use by the disabled or elderly users. A structural analysis is performed to confirm the structural safety of the wheelchair. Structure Mechanics Module of COMSOL Multiphysics is used as the analysis software. Based on the structural analysis of the whole frame and side frame, the maximum tensile stress and compressive stress are far below the acceptable level. This paper would be contributed to engineers who are interested in developing foldable wheel-chair and improve elderly and disabled users' life quality by expanding their right to mobility.

Yan-lin Wang et al. analyze kinematics and main muscle fatigue damages of lower extremity in Single crouch walking gait (SCWG). The lower extremity kinematics data of two walking gaits are collected through sensors and device, the collected kinematics data are processed by averaging and Fourier series function, the movement of musculoskeletal model of lower extremity is realized by driving of the joint motion functions. The biological characteristics of the main muscles of lower extremity are analyzed, and the effects of SCWG on the main muscle fatigue injury of lower extremity are obtained. The study results will fill in the gaps in the movement biomechanical characteristics (MBC) of lower extremity in SCWG and provide data for Rehabilitation Medical Technology (RMT) and development of rehabilitation equipment of lower extremity.

Overall, the special selection is to summarize recent discoveries and groundbreaking studies that will account for new challenge research in the broad and relevant area of Biomechanics in Medical Application ${ }^{1,2,8,12,13}$ in combination with biomedical imaging ${ }^{3-7,9-11,14}$ and relevant areas. Hope that the selected papers will 
provide the readers with interesting examples of current research on the most outstanding theoretical frameworks in biomechanics applied to medicine.

\author{
Guest Editors \\ Esteban Peña Pitarch \\ Universitat Politècnica de Catalunya (UPC), Spain \\ esteban.pena@upc.edu \\ Agnès Drochon \\ Centre National de la Recherche Scientifique (CNRS), France \\ agnes.drochon@utc.fr \\ Eddie Y. K. Ng \\ Nanyang Technological University, Singapore \\ MYKNG@ntu.edu.sg
}

\title{
References
}

1. Liu F, Ng EYK, Chen Zi, A special section on biological mechanics, J Mech Med Biol 15(6):1502002-1-1502002-3, 2015.

2. Liu F, Ng EYK, A special section on biological mechanics, $J$ Mech Med Biol 16(8):1602002-1-1602002-4, 2016.

3. Liu F, Ng EYK, A special section on biomedical imaging in diagnosis and treatment (Part 1), J Med Imag Health Inf 6(5):1209-1211, 2016.

4. Liu F, Ng EYK, A special section on biomedical imaging in diagnosis and treatment (Part 2), J Med Imag Health Inf 16(7):1670-1672, 2016.

5. Liu F, Ng EYK, A special section on biomedical imaging in diagnosis and treatment (Part 3), J Med Imag Health Inf 17(1):126-128, 2017.

6. Liu F, Ng EYK, A special section on methods and application in biomedical imaging (Part 1), J Med Imag Health Inf 7(5):919-921, 2017.

7. Liu F, Ng EYK, A special section on methods and application in biomedical imaging (Part 2), J Med Imag Health Inf 7(7):1522-1524, 2017.

8. Liu F, Ng EYK, A special section on biological mechanics, J Med Imag Health Inf 17(7):1702002-1-1702002-7, 2017.

9. Liu F, Ng EYK, A special section on methods and application in biomedical imaging (Part 3), J Med Imag Health Inf 8(1):1-4, 2018.

10. Gomez L, Ng EYK, A special section on methods and application in biomedical imaging (Part 1), J Med Imag Health Inf 8(7):1364-1367, 2018.

11. Gomez L, Ng EYK, A special section on methods and application in biomedical imaging (Part 2), J Med Imag Health Inf 8(8):1607-1610, 2018.

12. Pitarch EP, Drochon A, Ng EYK, A special selection on biological applications of mechanics, J Mech Med Biol 18(7):1802001-1-1802001-8, 2018.

13. Pitarch EP, Drochon A, Ng EYK, A special selection on biological applications of mechanics, J Mech Med Biol 18(8):1802002-1-1802002-8, 2018.

14. Gomez L, Ng EYK, A special section on methods and application in biomedical imaging (Part 3), J Med Imag Health Inf 9(1):43-46, 2019. 